\title{
A novel PTPN11 gene mutation bridges Noonan syndrome, multiple lentigines/LEOPARD syndrome and Noonan-like/multiple giant cell lesion syndrome
}

\author{
Anna Sarkozy ${ }^{1,2,4}$, Maria Gabriela Obregon ${ }^{3,4}$, Emanuela Conti ${ }^{1}$, Giorgia Esposito ${ }^{1,2}$, \\ Rita Mingarelli ${ }^{1}$, Antonio Pizzuti ${ }^{1,2}$ and Bruno Dallapiccola ${ }^{*, 1,2}$ \\ ${ }^{1}$ CSS Hospital, IRCCS, San Giovanni Rotondo and CSS-Mendel Institute, Rome, Italy; ${ }^{2}$ Department of Experimental \\ Medicine and Pathology, University 'La Sapienza', Rome, Italy; ${ }^{3}$ Hospital Italiano, Buenos Aires, Argentina
}

Noonan (NS) and multiple lentigines/LEOPARD syndromes (LS) have proved to be associated with distinct PTPN11 mutations. Noonan-like/multiple giant cell lesion syndrome (NLS) is a rare disease, characterised by short stature, facial dysmorphisms, congenital heart defect (CHD) and central giant cell lesions. PTPN11 gene mutations have been reported in a single NLS family and two sporadic patients. Here we report a patient with a complex phenotype progressing throughout the years from NS at birth towards LS and NLS. PTPN11 gene analysis disclosed a novel missense mutation (Ala461Thr) in exon 12, affecting the consensus sequence of the SHP2-active site. This observation joins together NS and LS to NLS into a unique genetic defect, broadening the clinical and molecular spectrum of PTPN11-related disorders.

European Journal of Human Genetics (2004) 12, 1069-1072. doi:10.1038/sj.ejhg.5201290

Published online 6 October 2004

Keywords: Noonan syndrome; PTPN11; multiple giant cell lesions

\section{Introduction}

Noonan syndrome (NS) is a common autosomal dominant multiple congenital anomaly disorder, characterised by short stature, craniofacial dysmorphisms and congenital heart defects (CHD), in particular pulmonary valve stenosis (PVS). ${ }^{1}$ NS shares several features with multiple lentigines (ML)/LEOPARD syndrome (LS), a distinct condition with short stature, CHD, ML, electrocardiographic conduction abnormalities and sensorineural deafness. ${ }^{2,3}$ NS and LS are allelic conditions, caused by different missense mutations in PTPN11 gene, on chromosome 12q24, even if genetic heterogeneity has been documented for both disorders. ${ }^{4-9}$

In 1974, Cohen et al described a patient with short stature, moderate developmental delay, facial dysmorph-

*Correspondence: Professor B Dallapiccola, Istituto CSS-Mendel, Viale Regina Margherita 261, 00198 Rome, Italy. Tel: 00390644160503; Fax: 00390644160548; E-mail: dallapiccola@css-mendel.it ${ }^{4}$ These two authors equally contributed to this work. Received 20 April 2004; revised 25 June 2004; accepted 6 July 2004 isms, short webbed neck, PVS, ML, pectus excavatum, cubitus valgus, generalised hypomineralisation, hearing loss, multiple central giant cell lesions (MGCL) of bones, joints and/or soft tissues, and considered this association as an entity sui generis. ${ }^{10}$ In 1991, based on the review of 14 patients, these authors designated this condition as Noonan-like/multiple giant cell lesion syndrome (NLS), and outlined a broad interindividual clinical variability, which was explained in part by incomplete reporting. ${ }^{11}$ They also distinguished NLS from NS, LS and cherubism, based on the specific location of MGCL. ${ }^{12}$ More recently, Bertola et al $^{13}$ reported on a family with typical NS and NLS features, and suggested allelism of these disorders. Molecular analysis in this family and in two sporadic NLS patients disclosed PTPN11 mutations previously associated with NS, substantiating the idea that NLS could be a variant of classic NS. ${ }^{5,14}$

Here we report on a young patient who manifested characteristics of NS at birth, progressing throughout the years towards LS and NLS. Molecular analysis identified a 
novel PTPN11 mutation, linking these phenotypes to a unique gene defect.

\section{Clinical report}

This white girl was the first child of healthy, nonconsanguineous parents. The father was 32 year old and the mother 28 years at the time of conception. Family history was unremarkable. The patient was born at 36 weeks gestation and was delivered by cesarean section after a uneventful pregnancy. Apgar score was 7 at $1 \mathrm{~min}$ and 8 at $5 \mathrm{~min}$. Birth weight was $3500 \mathrm{~g}$ ( $97 \mathrm{th}$ centile), length $46.5 \mathrm{~cm}$ (50th centile) and OFC $35 \mathrm{~cm}$ (90th centile). On examination, the infant showed an unusual face, cardiac murmur and hands and feet oedema. We evaluated the patient at the age of 1 month. Weight, length and OFC were within normal limits. Ocular hypertelorism, downslanting palpebral fissures, ptosis, blue-green irides, short nose with a wide base and depressed nasal bridge, low-set and posteriorly angulated ears with thick helices, micrognathia, short neck with excessive nuchal skin and widely spaced nipples were observed (Figure 1a). A few nevi, one café-au-lait spot on the abdomen, and a haemangioma on

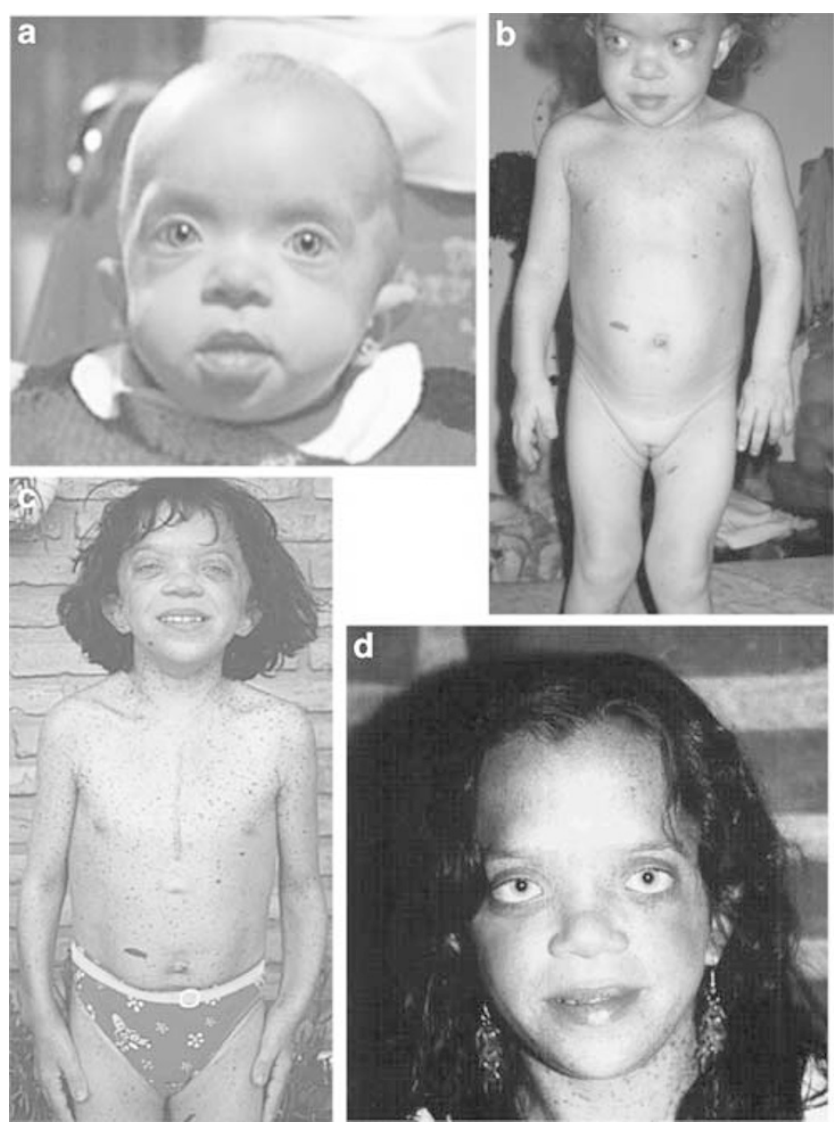

Figure 1 The proband at 1 month (a), at 4 years (b), at 10 years (c) and at 14 years of age (d), respectively. the back were noted. The echocardiogram was consistent with mild PVS, asymmetric septal hypertrophy, pulmonary artery branch stenosis and myxoid mitral valve. Abdominal ultrasound and chromosomes analysis were normal. At that time, a diagnosis of NS was suspected, based on a distinct NS facial gestalt (Figure 1a). At 7 months of age, bilateral severe sensorineural hearing loss and delayed psychomotor development mainly in the motor area was noticed, while ophthalmological evaluation was normal. At the age of 4 years, failure to thrive was reported (Figure 1b). At that time, she underwent cardiac catheterisation and pulmonary valve angioplasty and developed ML and multiple café-au-lait spots. For this reason, a diagnosis of LS was proposed. A cerebral CT scan disclosed mild dilatation of the lateral ventricles. At 10 years, PVS surgical correction with patch was performed. Short stature (height of $119 \mathrm{~cm},<3$ centile, - 2.5 SD) was reported, together with a Noonan-like facies, pectus excavatum, limited movement of elbows and wrists, Madelung deformity and enlarged ankles (Figure 1c). A skeletal survey showed generalised hypomineralisation with somewhat thickened trabeculae and cyst-like lesions in the mandible (Figure 2). Serum calcium, phosphate and PTH levels were normal. Osseous maturation was low normal (10 years at chronological age of 12 years). Thus, a diagnosis of NLS was raised. This patient has been followed for 14 years. At present, she presents incomplete development of secondary sexual characteristics with small breasts and hypoplastic external genitalia. Intelligence is normal, with a pleasant personality (Figure 1d).

The patient's parents were examined for major or minor dysmorphisms, cardiac and extracardiac anomalies, and found to be normal.

\section{Material and methods}

Informed consent was obtained for blood sampling the patient and unaffected parents. All PTPN11 gene coding region, including the 15 exons and exon-intron boundaries (GenBank Accession Number: NM_002834) were bidirectionally sequenced, using the Big Dye Terminator Abi Prism Sequencing Kit (Applied Biosystems, Foster City, CA, USA), and the ABI PRISM 3100 Genetic Analyser automated sequencer (Applied Biosystems, Foster City, CA, USA).

\section{Results}

PTPN11 gene analysis disclosed a missense heterozygous mutation in exon 12 . The $\mathrm{G} \rightarrow \mathrm{A}$ transition at position 1381 predicted an Ala461Thr substitution within the PTP domain. This residue is located in the consensus sequence (457-469 aa VHCSAGIGRTGTF) of the protein tyrosine signature motif involved in phosphate binding. The 

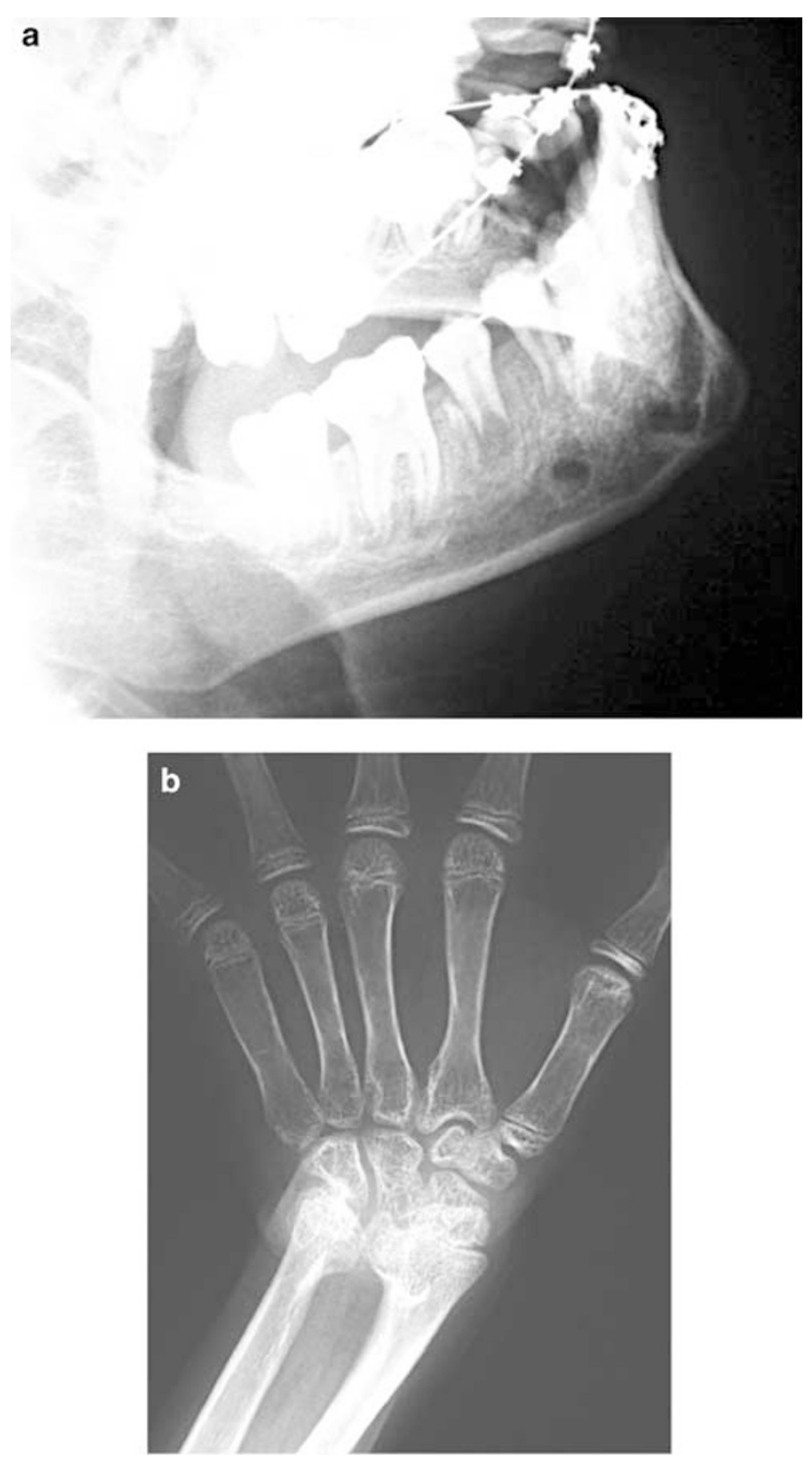

Figure 2 Rx ray films of the proband's mandible (a) and left hand (b). Note the presence of cyst-like lesions in the mandible and generalized hypomineralisation.

mutation was not identified in the unaffected parents and in any of 100 unaffected controls, and it has never been reported in any NS, LS and NLS patient. ${ }^{4-9}$

\section{Discussion}

PTPN11 gene mutations have been detected in association with NS, LS and NLS. Wide range clinical and molecular analyses have shown that NS and LS are clearly distinct conditions, with well-defined hallmark features and molecular defects. Although NLS patients display a number of distinct features, clinical and molecular overlaps with NS have suggested that these disorders are different manifestations of a unique clinical spectrum. ${ }^{5,13,14}$ In particular, the affected members of the family reported by Bertola et al carried an Asn308Ser mutation, recurrent among NS individuals, which has been correlated with typical NS features and PVS. ${ }^{5,8,13}$ Accordingly, affected family members presented with short stature, PVS and bleeding diathesis associated, in two of them, with giant cell lesions, which has raised the diagnosis of NLS. ${ }^{13}$ In addition, the same PTPN11 gene mutations, found in NS, have been detected also in two sporadic NLS individuals. ${ }^{14}$ Clinical features included several NS characteristics, such as facial dysmorphisms, pectus excavatum and PVS, associated with giant cell lesions in mandible and/or maxilla, confirmed at bioptic analysis, and osteopenia. ${ }^{14}$

The complex clinical presentation of our patient appears as a 'trait d'union' between NS, LS and NLS. Even if the partial overlap between NS and LS is well documented in early infancy, in this patient a nonquestionable NS phenotype was observed at birth. In fact, at that time, the presence of a unique café-au-lait spot and a few nevi did not suggested LS. Only with increasing age, the development of ML, sensorineural deafness and hypertrophic cardiomyopathy (HCM) prompted to the diagnosis of full-blown LS (Figure 1). Later on, detection of generalised hypomineralisation, cyst-like lesions in the mandible and low osseous maturation pointed to the diagnosis of NLS (Figure 2). This observation further underlines the lifetime variability observed in PTPN11-related phenotypes. The position of the mutated amino acid might partly explain this unusual 'changing' phenotype. In fact, the Ala461 residue is located in the consensus sequence, in close proximity to Gly464 and Thr468, commonly mutated in LS patients and associated with HCM. ${ }^{6,8-9}$

It is open to discussion if additional mutations in PTPN11 or, less likely, in other genes might be in charge of a clear-cut NLS phenotype, distinct from NS and LS, as suggested by Cohen and Gorlin. ${ }^{11}$ Molecular analysis of further NLS patients should be performed to investigate the genetic basis of the peculiar and heterogeneous clinical spectrum of NLS phenotype. Worthy of note, our patient showed a very striking resemblance with the patient of Cohen et $a^{11}$, which could argue for an identical molecular defect. Conversely, detection of the same mutation in different phenotypes, as for the Asn308Asp mutation, would suggest that modifier genes, epigenetic factors, second mutations or loss of heterozygosity of the wild PTPN11 allele could play a role in the phenotypic variability. In particular, central giant cell lesions could arise from additional somatic PTPN11 mutations. Interestingly, multiple giant cell granulomas have been reported also in association with Neurofibromatosis type 1 (NF1), and a novel splice mutation was found in one family affected by NF1 with central giant cell granulomas. ${ }^{15,16}$ 
PTPN11 and NF1 genes encode proteins with role in RAS signalling, and heterozygous somatic mutations have been detected in nonsyndromic haematologic malignances. ${ }^{17}$ One can speculate whether isolated giant cell lesions also arise from somatic mutations in either PTPN11 or NF1 genes.

In conclusion, we confirm that NLS is a PTPN11-related phenotype, characterised by central giant cell granulomas associated with a complex clinical presentation on the account of a distinct molecular defect. The novel PTPN11 mutation here reported joins together NS, LS and NLS, bridging these conditions into a unique clinical spectrum.

\section{Acknowledgements}

This study was supported in part by grants from the Italian Ministry of Health (RC 2004) and from the Italian Ministry of Instruction, University and Research (Grant 40\% to BD).

\section{References}

1 Allanson JE: Noonan syndrome. J Med Genet 1987; 24: 9-13.

2 Gorlin RJ, Anderson RC, Blaw M: Multiple lentigines syndrome. Am J Dis Child 1969; 117: 652-662.

3 Voron DA, Hatfield HH, Kalkhoff RK: Multiple lentigines syndrome. Case report and review of the literature. Am J Med 1976; 60: 447-456.

4 Tartaglia M, Mehler EL, Goldberg R et al: Mutations in PTPN11, encoding the protein tyrosine phosphatase SHP-2, cause Noonan syndrome. Nat Genet 2002; 30: 123-126.

5 Tartaglia M, Kalidas K, Shaw A et al: PTPN11 mutations in Noonan syndrome: molecular spectrum, genotype-phenotype correlation, and phenotypic heterogeneity. Am J Hum Genet 2002; 70: $1555-1563$.
6 Digilio MC, Conti E, Sarkozy A et al: Grouping of multiplelentigines/LEOPARD and Noonan syndromes on the PTPN11 gene. Am J Hum Genet 2002; 71: 389-394.

7 Conti E, Dottorini T, Sarkozy A et al: A novel PTPN11 mutation in LEOPARD syndrome. Hum Mut 2003; 21: 654

8 Sarkozy A, Conti E, Seripa D et al: Correlation between PTPN11 gene mutations and congenital heart defects in Noonan and LEOPARD syndromes. J Med Genet 2003; 40: 704-708.

9 Sarkozy A, Conti E, Digilio MC et al: Clinical and molecular analysis of 30 patients with multiple lentigines/LEOPARD syndrome. J Med Genet 2004; 41: e68.

10 Cohen Jr MM, Ruvalcaba RHA, Graham CB, Harrison MT, Morgan AF: A new syndrome simulating the Noonan syndrome, the Leopard syndrome, and hyperparathyroidism. Synd Ident 1974; 2: $14-17$.

11 Cohen Jr MM, Gorlin RJ: Noonan-like/multiple giant cell lesion syndrome. Am J Med Genet 1991; 40: 159-166.

12 Kaugars GE, Niamtu III J, Svirsky JA: Cherubism: diagnosis, treatment, and comparison with central giant cell granulomas and giant cell tumors. Oral Surg Oral Med Oral Pathol 1992; 73: 369-374.

13 Bertola DR, Kim CA, Pereira AC et al: Are Noonan syndrome and Noonan-like/multiple giant cell lesion syndrome distinct entities? Am J Med Genet 2001; 98: 230-234.

14 Lee JS, Fridrich KE, Reichenberger E et al: Phenotypic and genotypic carachterization of Noonan-like/multiple giant cell lesion syndrome. J Oral Maxillofac Surg 2002; 60 (Suppl): 57-58.

15 Ruggieri M, Pavone V, Polizzi A et al: Unusual form of recurrent giant cell granuloma of the mandible and lower extremities in a patient with neurofibromatosis type 1. Oral Surg Oral Med Oral Pathol Oral Radiol Endod 1999; 87: 67-72.

16 Krammer U, Wimmer K, Wiesbauer P et al: Neurofibromatosis 1: a novel NF1 mutation in an 11-year-old girl with a giant cell granuloma. J Child Neurol 2003; 18: 371-373.

17 Tartaglia M, Niemeyer CM, Fragale A et al: Somatic mutations in PTPN11 in juvenile myelomonocytic leukemia, myelodysplastic syndromes and acute myeloid leukemia. Nat Genet 2003; 34: $148-150$. 